九州大学学術情報リポジトリ

Kyushu University Institutional Repository

\title{
Time correlation functions in a similarity approximation for one-dimensional turbulence
}

Okamura, Makoto

Research Institute for Applied Mechanics, Kyushu University

Mori, Hazime

Research Institute for Applied Mechanics, Kyushu University

http://hdl. handle. net/2324/1910486

出版情報: Physical Review E. 79 (5)，pp.056312-1-056312-7，2009-05-26. American Physical Society

バージョン:

権利関係 : ๑2009 American Physical Society 


\title{
Time correlation functions in a similarity approximation for one-dimensional turbulence
}

\author{
Makoto Okamura and Hazime Mori \\ Research Institute for Applied Mechanics, Kyushu University, Kasuga 816-8580, Japan \\ (Received 27 November 2008; revised manuscript received 17 April 2009; published 26 May 2009)
}

\begin{abstract}
The projection operator formalism yields a time evolution equation for the time correlation function $U_{n}(t)$ of the chaotic modes of interest in terms of the memory function $\Gamma_{n}(t)$. On the assumption of similarity between $U_{n}(t)$ and $\Gamma_{n}(t)$, this equation leads to a closed equation for $U_{n}(t)$, which yields the asymptotic behavior of the time correlation function $U_{n}(t)$ and the corresponding power spectrum $I_{n}(\omega)$ analytically. Thus it turns out that the time correlation function takes the algebraic form $1 /\left(1+t^{2}\right)$ for $t \rightarrow 0$ as predicted previously, and can be classified into three decay forms for $t \rightarrow \infty$ according to the wave number $k_{n}$ : the exponential decay $e^{-t}$, the oscillatory exponential decay $e^{-t} \cos t$, and the oscillatory power-law decay $t^{-3 / 2} \cos t$. All the corresponding power spectra form a dual structure which is Lorentzian as $\omega \rightarrow 0$ and decays exponentially as $\omega \rightarrow \infty$. In the entire domain $0 \leq t<\infty$, solutions to the closed equation are quite consistent with the numerical results for small $k_{n}$, while they are consistent with those for large $k_{n}$, except for the phase. In the case that the integral time scale of $U_{n}(t)$ is equal to that of $\Gamma_{n}(t)$, the closed equation is identical to the direct interaction approximation equation for fluid turbulence in the limit $k_{n} \rightarrow \infty$.
\end{abstract}

DOI: 10.1103/PhysRevE.79.056312 PACS number(s): 47.27.Ak, 05.40.-a, 05.10.Gg, 05.45.-a

\section{INTRODUCTION}

The time correlation function or the power spectrum is one of the most important concepts for understanding chaotic motion such as turbulent flows. In a recent paper [1], it has been numerically shown for the chaotic KuramotoSivashinsky $(\mathrm{KS})$ equation that the time correlation function indicates the exponential decay $e^{-t}$ for $t \rightarrow \infty$ and it does the algebraic form $1 /\left(1+t^{2}\right)$ for $t \rightarrow 0$, representing the dual structure of chaos. This leads to the dual structure of the power spectra which is Lorentzian as $\omega \rightarrow 0$ and exponential as $\omega \rightarrow \infty$. These decay forms have been decided from the numerical results by using the best fit method. It is, therefore, interesting to derive analytically these decay forms for turbulence in general.

The purpose of the present paper is to clarify the time correlation function and the corresponding power spectrum, as well as their asymptotic forms, for one-dimensional turbulence in general by using the projection operator method. A closed equation for the time correlation function is necessary for this, and hence the closure problem of turbulence is inevitable [2].

There are few studies that evaluate theoretically the time correlation function or the power spectrum for turbulence [3-5], whereas there are many studies of phenomenological turbulence models [6]. The projection operator method presented here falls into the former category and is powerful in the context of the closure problem of the time correlation function because this method yields a very simple form of the time evolution equation for the time correlation function. In the projection operator formalism, some methods, such as the continued fraction expansion $[7,8]$, the mode-coupling theory $[9,10]$, and various other techniques $[11,12]$, have also been developed to evaluate the time correlation function, but they do not yield a practical estimate of the time correlation function in non-Markovian cases because of the existence of the closure problem [13].

The present paper is organized as follows. In Sec. II, we introduce a basic equation in one-dimensional turbulence and a time evolution equation of the time correlation function in the projection operator formalism. In Sec. III, a closure equation is derived from the time evolution equation under a similarity approximation (SA), and we discuss the appropriateness of this assumption. In Sec. IV, we solve numerically the closure equation and compare its solutions with the numerical solutions to the KS equation. In Sec. V, we solve analytically the closure equation and investigate the asymptotic behavior of the time correlation functions and the corresponding power spectra. In Sec. VI, we discuss the relation between the closure equation derived here and the direct interaction approximation (DIA) equation in threedimensional isotropic homogeneous turbulence.

\section{BASIC EQUATIONS}

We treat one-dimensional turbulence in general, the dynamics of which are governed by

$$
u_{t}=G\left(u, u_{x}, u_{x x}, \ldots\right),
$$

satisfying the periodic boundary condition $u(x, t)=u(x+L, t)$. Basic equation (1) is assumed to be invariant under time transformations, space transformations, and parity [14]. The $N$-truncated Fourier transform of Eq. (1) yields $N$ time evolution equations

$$
\frac{d \hat{u}_{n}(t)}{d t}=\hat{G}_{n}[\hat{\boldsymbol{u}}(t), \boldsymbol{k}], \quad n=1, \ldots, N,
$$

where $\hat{\boldsymbol{u}}(t) \equiv\left\{\hat{u}_{1}(t), \ldots, \hat{u}_{N}(t)\right\}, \boldsymbol{k} \equiv\left\{k_{1}, \ldots, k_{N}\right\}$, and the Fourier coefficient $\hat{u}_{n}(t)$, the wave number $k_{n}$, and $\hat{G}_{n}[\hat{\boldsymbol{u}}(t), \boldsymbol{k}]$ are defined as

$$
\hat{u}_{n}(t) \equiv \int_{0}^{L} u(x, t) e^{-i k_{n} x} d x, \quad k_{n} \equiv \frac{2 n \pi}{L},
$$

and 


$$
\hat{G}_{n}[\hat{\boldsymbol{u}}(t), \boldsymbol{k}] \equiv \int_{0}^{L} G\left(u, u_{x}, u_{x x}, \ldots\right) e^{-i k_{n} x} d x,
$$

respectively. Using the projection operator $\mathcal{P}$ defined as [15]

$$
\mathcal{P} f(\hat{\boldsymbol{u}}) \equiv \sum_{n=1}^{N} \frac{\left\langle f(\hat{\boldsymbol{u}}) \hat{u}_{n}\right\rangle}{\left\langle\hat{u}_{n} \hat{u}_{n}^{*}\right\rangle} \hat{u}_{n},
$$

where $\hat{u}_{n} \equiv \hat{u}_{n}(0), f(\hat{\boldsymbol{u}})$ is an arbitrary function of $\hat{\boldsymbol{u}}$, and the asterisk $*$ and the angular brackets $\langle\cdots\rangle$ denote the complex conjugation and the long-time average, respectively, we obtain the generalized Langevin equation

$$
\frac{d \hat{u}_{n}(t)}{d t}=-\int_{0}^{t} \Gamma_{n}(s) \hat{u}_{n}(t-s) d s+r_{n}(t),
$$

from Eq. (2) under the assumptions of statistical steadiness, statistical homogeneity, and statistical parity invariance [16]. Here,

$$
\begin{gathered}
\Gamma_{n}(t) \equiv-\frac{\left\langle\left[\Lambda r_{n}(t)\right] \hat{u}_{n}\right\rangle}{\left\langle\left|\hat{u}_{n}\right|^{2}\right\rangle}=\frac{\left\langle r_{n}(t) r_{n}^{*}\right\rangle}{\left\langle\left|\hat{u}_{n}\right|^{2}\right\rangle}, \\
r_{n}(t) \equiv e^{\mathcal{Q} \Lambda t} \mathcal{Q} \hat{G}_{n}(\hat{\boldsymbol{u}}, \boldsymbol{k}), \quad \mathcal{Q} \equiv 1-\mathcal{P},
\end{gathered}
$$

and

$$
\Lambda \equiv \sum_{n=1}^{N} \hat{G}_{n}(\hat{\boldsymbol{u}}, \boldsymbol{k}) \frac{\partial}{\partial \hat{u}_{n}} .
$$

The function $\Gamma_{n}(t)$ is called a memory function, bringing about the energy dissipation, and Eq. (4) is the fluctuationdissipation theorem of the second kind, which relates the memory function to the time correlation function $\left\langle r_{n}(t) r_{n}^{*}\right\rangle$ of the fluctuating motion $r_{n}(t)$. And the integral of the memory function is related to the eddy viscosity in the case that the wave number $k_{n}$ approaches zero [16].

By multiplying Eq. (3) by $\hat{u}_{n}^{*}$ and then averaging, we obtain the time evolution equation for the time correlation function $U_{n}(t) \equiv\left\langle\hat{u}_{n}(t) \hat{u}_{n}^{*}(0)\right\rangle$

$$
\frac{d U_{n}(t)}{d t}=-\int_{0}^{t} \Gamma_{n}(s) U_{n}(t-s) d s .
$$

It is important to note that Eq. (5) is not a closed but exact equation under the three assumptions.

We can obtain a closure equation from Eq. (5) by assuming a relation between $U_{n}(t)$ and $\Gamma_{n}(t)$. In order to ensure the validity of both the relation and the closure equation, we carry out direct numerical simulation (DNS) of the KS equation $[17,18]$

$$
u_{t}+u u_{x}+u_{x x}+u_{x x x x}=0,
$$

which is a typical example of one-dimensional turbulence, and a comparison is made between the time correlation function obtained from the KS equation and that from the closure equation. We evaluate numerically the time correlation function $U_{n}(t)$ for $0 \leq t \leq T_{m}$ as follows:



FIG. 1. The integral time scales $\tau_{n}^{(u)}$ (broken line) and $\tau_{n}^{(\gamma)}$ (solid line) as functions of $k_{n}$.

$$
U_{n}(t)=\left\langle\hat{u}_{n}(t) \hat{u}_{n}^{*}(0)\right\rangle=\frac{1}{M} \sum_{j=0}^{M-1} \hat{u}_{n}\left(t+T_{s}+j T_{m}\right) \hat{u}_{n}^{*}\left(T_{s}+j T_{m}\right),
$$

where the starting time is $T_{s}=1000$; the final time, $T_{f}=5$ $\times 10^{7}$; the maximum correlation time $T_{m}=40$; and the "ensemble" number, $M=\left(T_{f}-T_{s}\right) / T_{m} \approx 1.2 \times 10^{6}$, except for special cases. We have used a pseudospectral method with $N$ $=256$ for the spatial derivative and the fourth-order RungeKutta method with a time increment of 0.1 for the time evolution. The spatial period $L$ is chosen to be 500, which is sufficiently large for the KS equation to produce chaotic solutions.

\section{SIMILARITY APPROXIMATION EQUATION}

Let us assume the similarity between the time correlation function $U_{n}(t)$ and the memory function $\Gamma_{n}(t)$ as follows:

$$
Q_{n}(T) \equiv \frac{U_{n}\left(T \tau_{n}^{(u)}\right)}{U_{n}(0)}=\frac{\Gamma_{n}\left(T \tau_{n}^{(\gamma)}\right)}{\Gamma_{n}(0)},
$$

which is called a similarity approximation. Here the integral time scales $\tau_{n}^{(u)}$ and $\tau_{n}^{(\gamma)}$ are defined as

$$
\tau_{n}^{(u)} \equiv \frac{1}{U_{n}(0)} \int_{0}^{\infty} U_{n}(t) d t, \quad \tau_{n}^{(\gamma)} \equiv \frac{1}{\Gamma_{n}(0)} \int_{0}^{\infty} \Gamma_{n}(t) d t,
$$

and, $T$ is nondimensional time. Figure 1 shows $\tau_{n}^{(u)}$ and $\tau_{n}^{(\gamma)}$ as functions of $k_{n}$ obtained from DNS of the KS equation (6). The power spectrum $I_{n}(\Omega)$ is defined as

$$
I_{n}(\Omega) \equiv \frac{1}{\pi} \int_{0}^{\infty} Q_{n}(T) \cos (\Omega T) d T .
$$

Substituting Eq. (7) into Eq. (5), we obtain a closure equation

$$
\frac{d Q_{n}(T)}{d T}=-\int_{0}^{T / \widetilde{\tau}_{n}} Q_{n}(S) Q_{n}\left(T-\widetilde{\tau}_{n} S\right) d S,
$$

where we have used $\Gamma_{n}(0)=1 /\left(\tau_{n}^{(u)} \tau_{n}^{(\gamma)}\right)$ and defined

$$
\widetilde{\tau}_{n} \equiv \frac{\tau_{n}^{(\gamma)}}{\tau_{n}^{(u)}},
$$

which satisfies $0<\widetilde{\tau}_{n} \leq 1$. The closure equation (9) is a nonMarkovian nonlinear evolution equation for $Q_{n}(T)$, which 

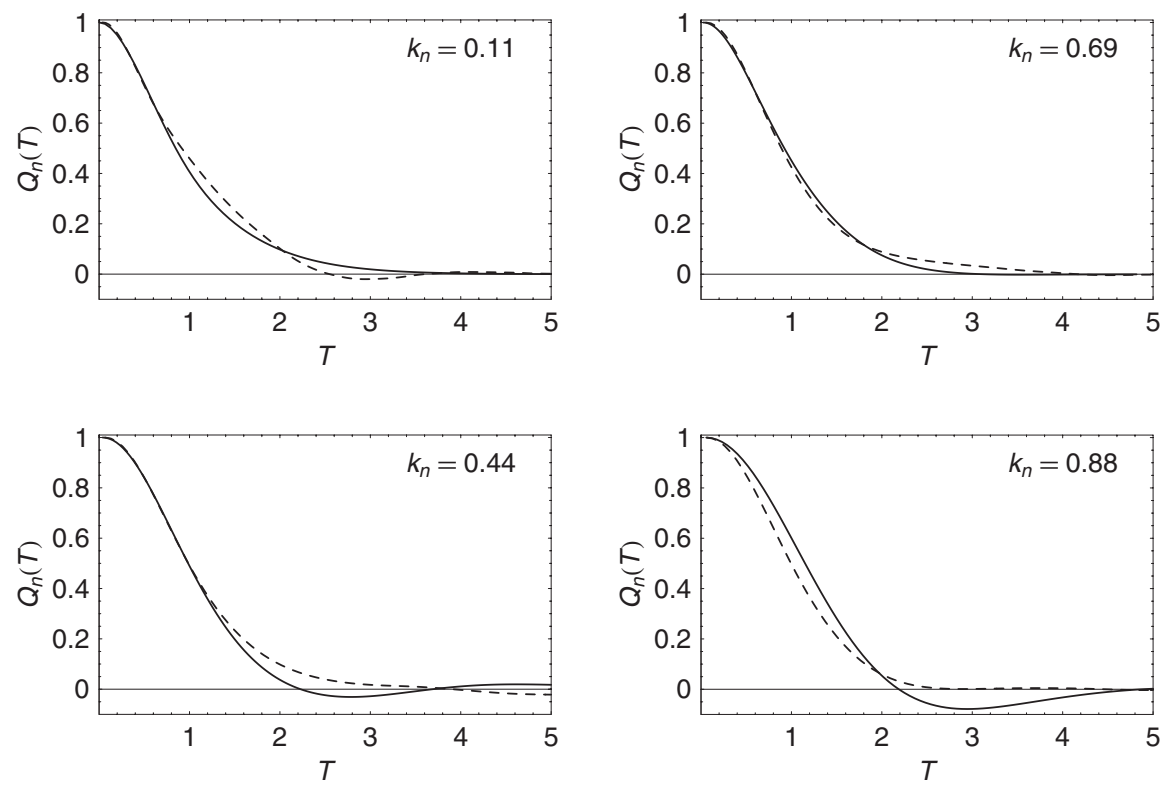

FIG. 2. The scaled and normalized time correlation function $U_{n}\left(T \tau_{n}^{(u)}\right) / U_{n}(0)$ (solid line) and memory function $\Gamma_{n}\left(T \tau_{n}^{(\gamma)}\right) / \Gamma_{n}(0)$ (broken line) as functions of $T$ in the cases $k_{n}=0.11,0.44,0.69$, and 0.88 .

has been derived under similarity approximation (7). We, therefore, refer to Eq. (9) as the SA equation.

Figure 2 shows the scaled and normalized time correlation functions $U_{n}\left(T \tau_{n}^{(u)}\right) / U_{n}(0)$ and memory functions $\Gamma_{n}\left(T \tau_{n}^{(\gamma)}\right) / \Gamma_{n}(0)$ obtained from DNS of the KS equation (6) in the cases $k_{n}=0.11,0.44,0.69$, and 0.88 . Because the difference between them is small in all the four cases, the similarity approximation (7) is justified for the KS equation (6). The time correlation function $U_{n}(t)$ for $0 \leq t \leq T_{m}=100$ has been used only in the case $k_{n}=0.11$ because $U_{n}(t)$ for $0 \leq t$ $\leq T_{m}=40$ corresponds to $U_{n}\left(T \tau_{n}^{(u)}\right) / U_{n}(0)$ for $0 \leq T \leq 2.62$ in the case $k_{n}=0.11$, which is not enough for this figure with the range $0 \leq T \leq 5$.

We now explain the reason why the similarity approximation (7) is justified. The time correlation function $U_{n}(t)$ is related to the slowly varying motion for small $k_{n}$ and to the rapidly varying motion for large $k_{n}$, while the memory function $\Gamma_{n}(t)$ is related to the fluctuating motion, and hence there seems not to be the relation between the time correlation function and the memory function. However, we point out a possibility that there is a relation between them as follows. First, the $n$th mode $\hat{u}_{n}(t)$ indicates simple motion with one characteristic time scale $\tau_{n}^{(u)}$, which is shown in Fig. 1, while the chaotic motion $f_{n}(t) \stackrel{n}{\equiv} d \hat{u}_{n}(t) / d t$ includes the nonlinear term such as

$$
N_{n}(t) \equiv-\frac{i}{L} \sum_{m=-N}^{N} k_{m} \hat{u}_{n-m}(t) \hat{u}_{m}(t),
$$

which brings about complex motion with more than two characteristic time scales. The time correlation function of the complex motion $f_{n}(t)$ is shown in Fig. 3, which indicates that there are two kinds of motion: the slowly varying motion and the fluctuating motion. Hence, all the time correlation functions $U_{n}(t)$ of $\hat{u}_{n}(t)$ are almost similar in shape because their motions are simple, while the time correlation functions $F_{n}(t) \equiv\left\langle f_{n}(t) f_{n}^{*}(0)\right\rangle$ are not similar in shape. This similarity is verified by comparing the scaled and normalized time correlation functions $U_{n}\left(T \tau_{n}^{(u)}\right) / U_{n}(0)$ shown in Fig. 2 . Second, the normalized memory functions $\Gamma_{n}(t) / \Gamma_{n}(0)$ have the same forms because their integral time scales are nearly independent of $k_{n}$, which is shown in Fig. 1. Third, the time correlation function $U_{n}(t)$ is similar to the memory function $\Gamma_{n}(t)$ for large $k_{n}$ because the rapidly varying motion $\hat{u}_{n}(t)$ for large $k_{n}$ is quite similar to the fluctuating motion $r_{n}(t)$. Therefore, we can expect the similarity approximation (7).

Furthermore, we discuss a strong connection between the similarity approximation (7) and the fluctuation-dissipation theorem of the second kind (4). The projection operator method divides the chaotic motion $f_{n}(t)=d \hat{u}_{n}(t) / d t$ into two parts: the slowly varying motion $s_{n}(t)$ and the "fluctuating" motion $r_{n}(t)$ [16]. In the case that $k_{n}$ is a small value such as 0.06 , the time correlation function $F_{n}(t)=\left\langle f_{n}(t) f_{n}^{*}(0)\right\rangle$ has two characteristic time scales as shown in Fig. 3. This figure shows that the slowly varying motion can be successfully subtracted by using the projection operator because the time correlation function $R_{n}(t) \equiv\left\langle r_{n}(t) r_{n}^{*}(0)\right\rangle$ does not include the slowly varying motion, which demonstrates that $r_{n}(t)$ is the true fluctuating motion, as expected. If the subtraction is not successful, the "fluctuating" motion $r_{n}(t)$ includes certain slowly varying motion, and hence the similarity approximation and the fluctuation-dissipation theorem are broken.

To end this section, we make comments about the relation between $\tau_{n}^{(\gamma)}$ and $\tau_{n}^{(u)}$. The integral time scale $\tau_{n}^{(\gamma)}$ denotes the

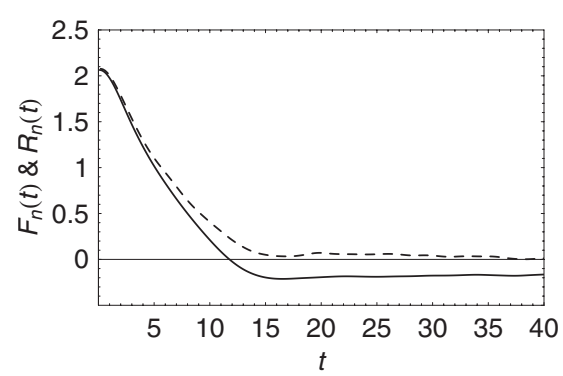

FIG. 3. The time correlation functions $F_{n}(t)=\left\langle f_{n}(t) f_{n}^{*}(0)\right\rangle$ (solid line) and $R_{n}(t)=\left\langle r_{n}(t) r_{n}^{*}(0)\right\rangle$ (broken line) in the case $k_{n}=0.06$. 

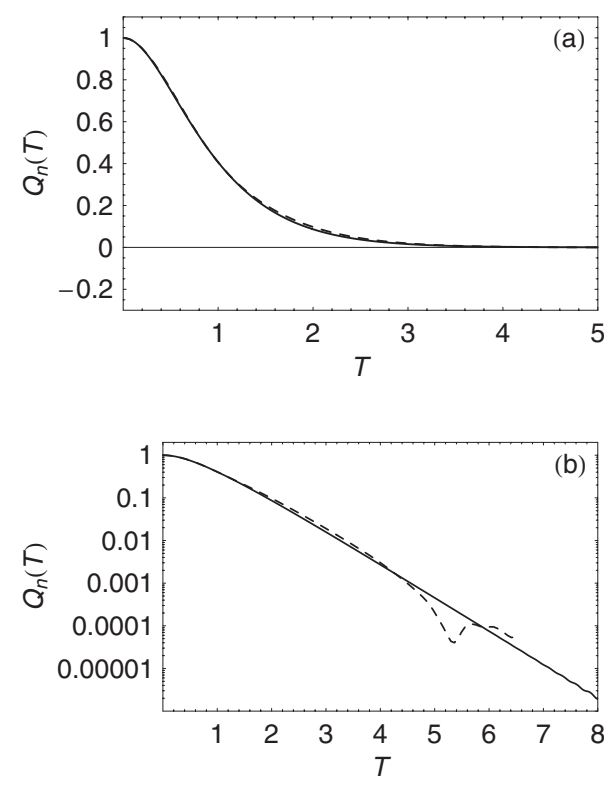

FIG. 4. (a) Linear-linear and (b) semilogarithmic plots of the time correlation functions $Q_{n}(T)$ for $\widetilde{\tau}_{n}=0.36\left(k_{n}=0.11\right):-$, the SA equation (9); and - - , DNS. $T_{m}=100$ and $M=2.3 \times 10^{7}$.

characteristic time of the fluctuating motion $r_{n}(t)$, which is nearly independent of $k_{n}$ and is related to the minimum time scale of the chaotic motion, while $\tau_{n}^{(u)}$ denotes the characteristic time of $\hat{u}_{n}(t)$, which depends on $k_{n}$. Hence, $\tau_{n}^{(\gamma)} \leq \tau_{n}^{(u)}$, which is supported by the numerical result shown in Fig. 1.

\section{NUMERICAL SOLUTIONS TO THE SA EQUATION}

We compare the time correlation functions obtained numerically from the SA equation (9) and those from the KS equation (6) in the cases $\widetilde{\tau}_{n}=0.36$ and 0.88 , which correspond to $k_{n}=0.11$ and 0.88 , respectively. In DNS of the KS equation, we have used the maximum correlation time $T_{m}$ $=100$ and the ensemble number $M=2.3 \times 10^{7}$ for $\widetilde{\tau}_{n}=0.36$, and $T_{m}=40$ and $M=1.7 \times 10^{8}$ for $\widetilde{\tau}_{n}=0.88$. These values are chosen to be larger than those in other simulations because they are necessary to make a detailed comparison between the time correlation functions obtained from the SA equation and from the KS equation.

Figure 4 shows the linear-linear and semilogarithmic plots of the time correlation functions $Q_{n}(T)$ in the case $\widetilde{\tau}_{n}=0.36$, which corresponds to $k_{n}=0.11$. The solution to the SA equation (9) is quite consistent with the result obtained from DNS of the KS equation (6), and hence there is no graphical difference between them in the linear-linear plot. The semilogarithmic plot indicates that the time correlations decay exponentially. The difference between them for $T \gtrsim 4.5$ is due to the lack of the ensemble number $M$. The DNS data (broken line) breaks off at $T=6.6$ because $U_{n}(t)$ for $0 \leq t \leq T_{m}=100$ corresponds to $U_{n}\left(T \tau_{n}^{(u)}\right) / U_{n}(0)$ for $0 \leq T \leq 6.6$ in the case $\widetilde{\tau}_{n}=0.36$.

Figure 5 shows the linear-linear and semilogaritmic plots of the time correlation functions $Q_{n}(T)$ in the case $\widetilde{\tau}_{n}=0.88$, which corresponds to $k_{n}=0.88$. The solution to the SA equation (9) is consistent with the result obtained from DNS of
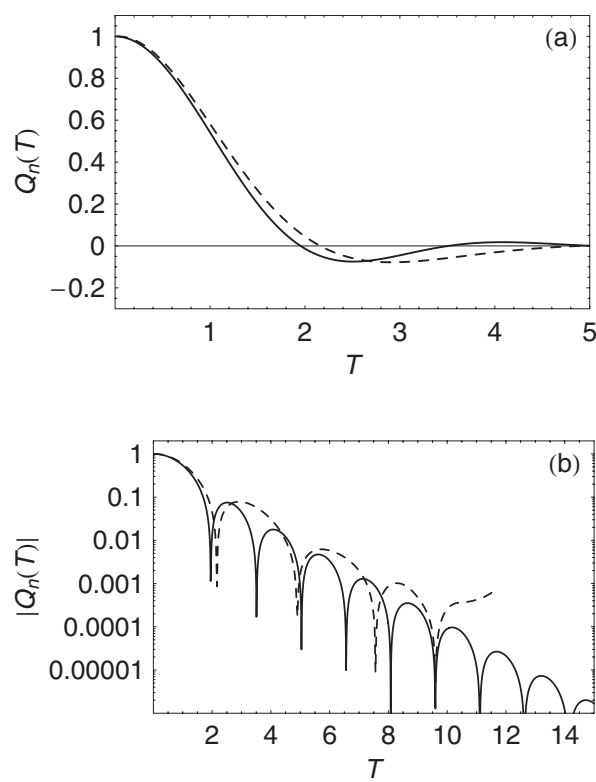

FIG. 5. (a) Linear-linear and (b) semilogarithmic plots of the time correlation functions $Q_{n}(T)$ for $\widetilde{\tau}_{n}=0.88\left(k_{n}=0.88\right)$ : - , the SA equation (9); and - - DNS. $T_{m}=40$ and $M=1.7 \times 10^{8}$.

the KS equation (6), except for the phase. This phase difference is nearly twice, but the reason for this is uncertain at the present. The semilogarithmic plot indicates that the time correlations decay exponentially with oscillation. The DNS data (broken line) breaks off at $T=11.5$ because $U_{n}(t)$ for $0 \leq t$ $\leq T_{m}=40$ corresponds to $U_{n}\left(T \tau_{n}^{(u)}\right) / U_{n}(0)$ for $0 \leq T \leq 11.5$ in the case $\widetilde{\tau}_{n}=0.88$.

\section{ANALYTICAL SOLUTIONS TO THE SA EQUATION}

We now investigate the time correlation functions $Q_{n}(T)$ for three asymptotic cases, $T \ll \widetilde{\tau}_{n}, T \rightarrow \infty$, and $\widetilde{\tau}_{n} \ll 1$, by solving the SA equation (9) analytically.

\section{A. Time correlation function $Q_{n}(T)$ for $T \ll \tau_{n}$}

The asymptotic behavior of the time correlation function is analytically investigated for $t \rightarrow 0$. Because each of $U_{n}(t)$ and $\Gamma_{n}(t)$ has a peak at $t=0$ for the KS equation (6) as shown in Fig. 2, the similarity approximation (7) is very accurate in this case.

In the case $T \ll \widetilde{\tau}_{n}$, Eq. (9) is reduced to

$$
\frac{d Q_{n}(T)}{d T} \approx-\frac{\left[Q_{n}(T)\right]^{2} T}{\widetilde{\tau}_{n}}
$$

and its solution is

$$
Q_{n}(T)=\frac{2 \widetilde{\tau}_{n}}{T^{2}+2 \widetilde{\tau}_{n}} \text { for } T \ll \widetilde{\tau}_{n},
$$

which is called the algebraic form and is identical to the form in Ref. [1].

We now compare the analytical result (10) for $\widetilde{\tau}_{n}=0.44$ with the result obtained from DNS of the KS equation (6) for $k_{n}=0.69$, corresponding to $\widetilde{\tau}_{n}=0.44$. Figure 6 shows that al- 


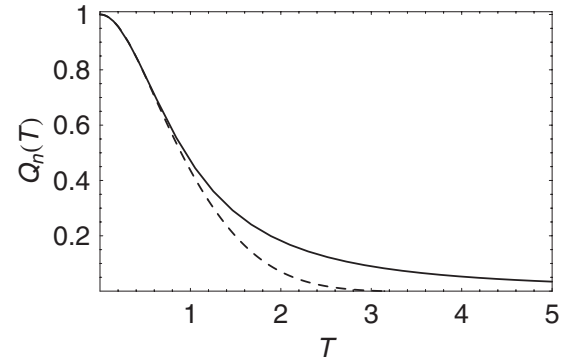

FIG. 6. The time correlation function $Q_{n}(T)$ for $\tilde{\tau}_{n}=0.44$ $\left(k_{n}=0.69\right)$ : — algebraic form (10); and --- , DNS.

gebraic form (10) is consistent with the numerical result for $T \leqq \widetilde{\tau}_{n}=0.44$. The well-known Gaussian form $\exp [$ $\left.-T^{2} /\left(2 \widetilde{\tau}_{n}\right)\right]$ is also consistent with the numerical result for small $T$. In order to make clearer the difference between algebraic form (10) and the Gaussian form, we compare the corresponding power spectra. Substituting Eq. (10) into Eq. (8), we obtain the power spectrum

$$
I_{n}(\Omega)=\sqrt{\frac{\tilde{\tau}_{n}}{2}} \exp \left(-\sqrt{2 \widetilde{\tau}_{n}} \Omega\right) \text { for } \Omega \gg 1 / \widetilde{\tau}_{n},
$$

which is called the exponential spectrum [1], while the power spectrum corresponding to the Gaussian form is the Gaussian spectrum $\exp \left(-\Omega^{2} \widetilde{\tau}_{n} / 2\right)$. Figure 7 shows that the exponential spectrum (11) is consistent with the numerical result for $\Omega \gtrsim 1 / \widetilde{\tau}_{n}=2.3$, including the value of the nondimensional characteristic time $\sqrt{2 \widetilde{\tau}_{n}}$ in Eq. (11). Apparently, the Gaussian spectrum is inconsistent with the numerical result. Therefore, we conclude that the time correlation function is algebraic form (10) for small $T$ and the corresponding power spectrum is the exponential decay (11) for large $\Omega$. It is important to note that the asymptotic power spectrum becomes the exponential spectrum for large $\Omega$ also in the stochastic frequency modulation model [1] because this fact may suggest that the exponential spectrum is a universal decay form.

\section{B. Time correlation function $Q_{n}(T)$ as $T \rightarrow \infty$}

Next, we investigate the asymptotic decay form of the time correlation function $Q_{n}(T)$ as $T \rightarrow \infty$. Because it is difficult to obtain asymptotic solutions to Eq. (9) as $T \rightarrow \infty$, we consider the Fourier-Laplace transform of Eq. (9) and seek

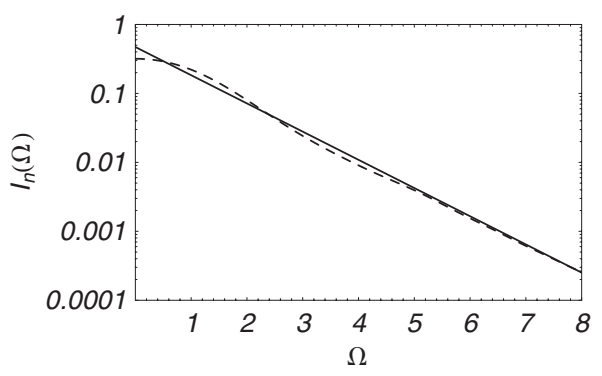

FIG. 7. Semilogarithmic plot of the power spectrum $I_{n}(\Omega)$ for $\widetilde{\tau}_{n}=0.44\left(k_{n}=0.69\right):-$, the exponential spectrum $(11)$; and --- , DNS. its solutions as $\Omega \rightarrow 0$. Denoting the Fourier-Laplace transforms of $Q_{n}(T), U_{n}(t)$, and $\Gamma_{n}(t)$ by

$$
\begin{gathered}
\hat{Q}_{n}(\Omega)=\int_{0}^{\infty} Q_{n}(T) e^{-i \Omega T} d T, \\
\hat{U}_{n}(\omega)=\int_{0}^{\infty} U_{n}(t) e^{-i \omega t} d t,
\end{gathered}
$$

and

$$
\hat{\Gamma}_{n}(\omega)=\int_{0}^{\infty} \Gamma_{n}(t) e^{-i \omega t} d t
$$

respectively $[1,19]$, we now assume that $\hat{U}_{n}(\omega)$ and $\hat{\Gamma}_{n}(\omega)$ are similar in shape near $\omega=0$;

$$
\hat{Q}_{n}(\Omega) \equiv \frac{\hat{U}_{n}\left(\Omega / \tau_{n}^{(u)}\right)}{\hat{U}_{n}(0)}=\frac{\hat{\Gamma}_{n}\left(\Omega / \tau_{n}^{(\gamma)}\right)}{\hat{\Gamma}_{n}(0)}, \quad \Omega \rightarrow 0,
$$

which corresponds to the similarity approximation (7). The power spectrum $I_{n}(\omega)$ can be expressed as

$$
I_{n}(\Omega)=\frac{1}{\pi} \operatorname{Re}\left[\hat{Q}_{n}(\Omega)\right],
$$

using Eqs. (8) and (12). The Fourier-Laplace transform of (9) yields

$$
\hat{Q}_{n}(\Omega) \hat{Q}_{n}\left(\Omega \widetilde{\tau}_{n}\right)+i \Omega \hat{Q}_{n}(\Omega)-1=0 \text { for } \Omega \rightarrow 0 .
$$

We further assume that the solution to Eq. (14) has the form of a Padé approximant for $\Omega \rightarrow 0$ as follows [20]:

$$
\hat{Q}_{n}(\Omega)=\frac{1+b_{n}^{(2)} \Omega^{2}}{1+a_{n}^{(2)} \Omega^{2}+a_{n}^{(4)} \Omega^{4}}+i \frac{b_{n}^{(1)} \Omega+b_{n}^{(3)} \Omega^{3}}{1+c_{n}^{(2)} \Omega^{2}+c_{n}^{(4)} \Omega^{4}} .
$$

The application of the Padé approximant to $\hat{Q}_{n}(\Omega)$ is natural because the Fourier-Laplace transform of (5) and the Taylor approximation to $\hat{\Gamma}_{n}(\omega)$ at $\omega=0$ yield the Padé approximant to $\hat{U}_{n}(\omega)[1]$. It follows from Eqs. (13) and (15) that the power spectrum is Lorentzian:

$$
I_{n}(\Omega)=\frac{1}{\pi} \frac{1}{1+a_{n}^{(2)} \Omega^{2}} \text { for } \Omega \rightarrow 0 .
$$

Substituting Eq. (15) into Eq. (14) and expanding in power series at $\Omega=0$ to fifth order, we obtain the coefficients, $a_{n}^{(i)}$, $b_{n}^{(i)}$, and $c_{n}^{(i)}$, as functions of $\widetilde{\tau}_{n}$. Using Eqs. (13) and (15) and the inverse transform of Eq. (8), we obtain the time correlation function

$$
Q_{n}(T)=\frac{2}{\pi} \int_{0}^{\infty} \frac{1+b_{n}^{(2)} \Omega^{2}}{1+a_{n}^{(2)} \Omega^{2}+a_{n}^{(4)} \Omega^{4}} \cos (\Omega T) d \Omega .
$$

We can evaluate integral (16) with the residue theorem separately in the following five cases, according to the value of $\tilde{\tau}_{n}$.

In the case $0<\tilde{\tau}_{n}, 0.37$, we obtain from Eq. (16) the time correlation function 


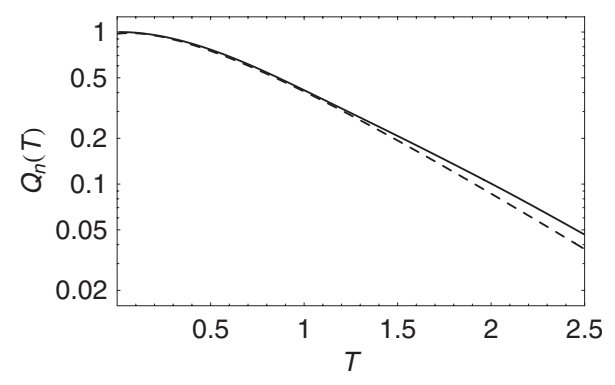

FIG. 8. The time correlation function $Q_{n}(T)$ for $\widetilde{\tau}_{n}=0.36\left(k_{n}\right.$ =0.11): - , Eq. (17); and --- , DNS.

$$
\begin{aligned}
Q_{n}(T) & =A_{n}^{(-)} e^{-\beta_{n}^{(-)} T}+A_{n}^{(+)} e^{-\beta_{n}^{(+)} T} \\
& \approx A_{n}^{(-)} e^{-\beta_{n}^{(-)} T} \text { for } T \rightarrow \infty
\end{aligned}
$$

where $A_{n}^{( \pm)}$and $\beta_{n}^{( \pm)}\left(\beta_{n}^{(+)} \geq \beta_{n}^{(-)}\right)$are known positive functions of $\widetilde{\tau}_{n}$. Figure 8 shows that the exponential decay (17) is consistent with the numerical result for large $T$ where $\widetilde{\tau}_{n}$ $=0.36\left(k_{n}=0.11\right)$.

In the case $0.37<\widetilde{\tau}_{n} \leq 0.84$, we obtain from Eq. (16) the time correlation function

$$
Q_{n}(T)=A_{n} e^{-\beta_{n} T} \cos \left(\alpha_{n} T+\theta_{n}\right) \text { for } T \rightarrow \infty,
$$

where $A_{n}, \beta_{n}, \alpha_{n}$, and $\theta_{n}$ are known functions of $\tilde{\tau}_{n}$. Figure 9 shows that the exponential decay with oscillation (19) is consistent with the numerical result for large $T$ where $\widetilde{\tau}_{n}$ $=0.44\left(k_{n}=0.69\right)$, except for the phase $\theta_{n}$.

In the case $0.84<\widetilde{\tau}_{n} \leq 0.85$, Eq. (16) yields a time correlation as in Eq. (17) and in the case $0.85<\widetilde{\tau}_{n}<1$ integral (16) is divergent, while the numerical solutions to the SA equation (9) suggest the exponential decay with oscillation (19) in the case $0.84<\widetilde{\tau}_{n}<1$ as shown in Fig. 5. This discrepancy occurs because the Padé approximant (15) is not appropriate for $0.84<\widetilde{\tau}_{n}<1$, and higher-order Padé approximants may improve the analytical solutions for $0.84<\widetilde{\tau}_{n}$ $<1$.

In the case $\widetilde{\tau}_{n}=1$, we obtain an exact solution,

$$
\hat{Q}_{n}(\Omega)=-i \frac{\Omega}{2}+\sqrt{1-\left(\frac{\Omega}{2}\right)^{2}}
$$

to Eq. (14), which leads to the time correlation function

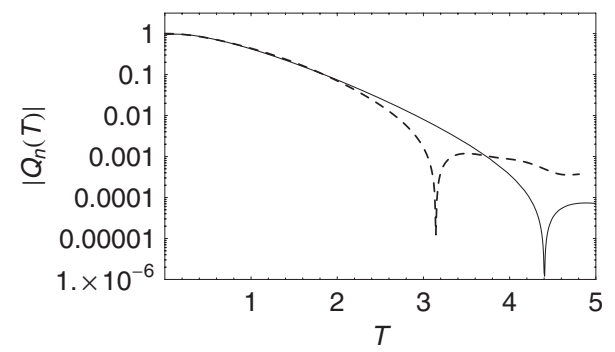

FIG. 9. The absolute value of $Q_{n}(T)$ for $\widetilde{\tau}_{n}=0.44\left(k_{n}=0.69\right)$ : - , Eq. (19); and - - -, DNS.

$$
Q_{n}(T)=\frac{1}{T} J_{1}(2 T) \approx \frac{T^{-3 / 2}}{\sqrt{\pi}} \cos \left(2 T-\frac{3 \pi}{4}\right) \text { for } T \rightarrow \infty
$$

where $J_{1}(T)$ is the Bessel function of the first kind. This equation indicates that the time correlation function $Q_{n}(T)$ is the oscillatory power-law decay as $T \rightarrow \infty$.

In summary, the time correlation functions $Q_{n}(T)$ can be classified into three decay forms as $T \rightarrow \infty$ according to $\widetilde{\tau}_{n}$ (or $k_{n}$ ) as follows:

$$
Q_{n}(T) \propto \begin{cases}e^{-\beta_{n}^{(-)} T} & \text { for } 0<\tilde{\tau}_{n} \leq 0.37 \\ e^{-\beta_{n} T} \cos \left(\alpha_{n} T+\theta_{n}\right) & \text { for } 0.37 \leq \tilde{\tau}_{n} \leq 0.84 \\ - & \text { for } 0.84<\tilde{\tau}_{n}<1 \\ \frac{T^{-3 / 2}}{\sqrt{\pi}} \cos \left(2 T-\frac{3 \pi}{4}\right) & \text { for } \tilde{\tau}_{n}=1,\end{cases}
$$

where "_-" means that the analytical solutions are inconsistent with the numerical solutions for $0.84<\widetilde{\tau}_{n}<1$, while in the previous paper [1],

$$
Q_{n}(T) \propto e^{-\beta_{n}^{(-)} T} \text { for } 0<\widetilde{\tau}_{n} \leq 1 .
$$

However, the power spectrum $I_{n}(\Omega)$ becomes Lorentzian,

$$
I_{n}(\Omega)=\frac{1}{\pi} \frac{1}{1+a_{n}^{(2)} \Omega^{2}} \text { for } \Omega \rightarrow 0,
$$

in all of these four cases. Therefore, the dynamic structures are characterized by the Lorentzian peaks for $\Omega \rightarrow 0$ and the exponential spectra (11) for $\Omega \rightarrow \infty$, as predicted previously [1]. Note that the classification of the decay form is expected for one-dimensional turbulence in general because the present analysis is not restricted to the KS equation (6).

\section{Time correlation function $Q_{n}(T)$ for $\widetilde{\tau}_{n} \ll 1$}

In the case $\widetilde{\tau}_{n} \ll 1$, the SA equation (9) yields the solution

$$
Q_{n}(T)=e^{-T},
$$

which is compatible with Eq. (18) because $\beta_{n}^{(-)} \rightarrow 1$ as $\tilde{\tau}_{n}$ $\rightarrow 0$. Because $\widetilde{\tau}_{n} \rightarrow 0$ corresponds to $k_{n} \rightarrow 0$, Eq. (21) is consistent with the solution to Eq. (5) under the Markov approximation, i.e., $\Gamma_{n}(t) \propto \delta(t)[21]$.

\section{SUMMARY AND CONCLUDING REMARKS}

We have derived the closure equation, called the SA equation (9), from the one-dimensional equation (1) under the similarity assumption (7). In order to ensure the validity of the SA equation, its solutions have been numerically compared with the solutions to the KS equation (6). By solving analytically the SA equation, we have classified the time correlation function $U_{n}(t)$ into three decay forms for $t \rightarrow \infty$ according to the wave number $k_{n}$ : the exponential decay $e^{-t}$, the oscillatory exponential decay $e^{-t} \cos t$, and the oscillatory power-law decay $t^{-3 / 2} \cos t$. All the corresponding power spectra $I_{n}(\omega)$ become Lorentzian as $\omega \rightarrow 0$ and decay expo- 
nentially as $\omega \rightarrow \infty$, in agreement with the previous prediction [1].

To end this paper, we make comments about the relation between the SA equation (9) in one-dimensional homogeneous turbulence and the DIA equation in three-dimensional isotropic homogeneous turbulence. The SA equation with $\widetilde{\tau}_{n}=1$ is identical to the DIA equation for $k_{n} \rightarrow \infty$ [3]. Because $k_{n} \rightarrow \infty$ is expected to correspond to $\widetilde{\tau}_{n} \rightarrow 1$, the two approximations are identical in the case $k_{n} \rightarrow \infty$. The DIA equation does not yield the correct characteristic time scale of turbu- lence because of the sweeping effect on Eulerian time correlations [22]. This effect obscures small scale motion and hence, in the case $k_{n} \rightarrow \infty$, the solution may be independent of the details of the equation. Therefore the SA equation is identical to the DIA equation for $k_{n} \rightarrow \infty$. Another example is the Brownian motion with retardation in viscous resistance due to the hydrodynamic backflow effect [19]. Because the backflow obscures the Brownian motion, the time correlation indicates a power-law decay $t^{-3 / 2}$ similar to the present result (20), except for the oscillatory part.
[1] H. Mori and M. Okamura, Phys. Rev. E 76, 061104 (2007).

[2] P. A. Davidson, Turbulence: An Introduction for Scientists and Engineers (Oxford University Press, Oxford, 2004).

[3] R. H. Kraichnan, J. Fluid Mech. 5, 497 (1959).

[4] Y. Kaneda, Phys. Fluids 29, 701 (1986).

[5] S. Kida and S. Goto, J. Fluid Mech. 345, 307 (1997).

[6] S. B. Pope, Turbulent Flows (Cambridge University Press, Cambridge, 2000).

[7] H. Mori, Prog. Theor. Phys. 34, 399 (1965).

[8] D. Daems, S. Grossmann, V. S. L'vov, and I. Procaccia, Phys. Rev. E 60, 6656 (1999).

[9] K. Kawasaki, Ann. Phys. (N.Y.) 61, 1 (1970).

[10] T. Yamaguchi and F. Hirata, J. Chem. Phys. 117, 2216 (2002).

[11] U. Balucani and M. Zoppi, Dynamics of the Liquid State (Oxford University Press, New York, 1994).

[12] J. P. Boon and S. Yip, Molecular Hydrodynamics (McGrawHill, New York, 1980).

[13] U. Balucani, M. H. Lee, and V. Tognetti, Phys. Rep. 373, 409 (2003).
[14] U. Frisch, Turbulence: The Legacy of A. N. Kolmogorov (Cambridge University Press, Cambridge, 1995).

[15] H. Mori and H. Fujisaka, Phys. Rev. E 63, 026302 (2001).

[16] M. Okamura, Phys. Rev. E 74, 046210 (2006).

[17] Y. Kuramoto, Chemical Oscillations, Waves, and Turbulence (Springer-Verlag, Berlin, 1984).

[18] M. C. Cross and P. C. Hohenberg, Rev. Mod. Phys. 65, 851 (1993).

[19] R. Kubo, M. Toda, and N. Hashitsume, Statistical Physics II: Nonequilibrium Statistical Mechanics (Springer-Verlag, Berlin, 1991).

[20] C. M. Bender and S. A. Orszag, Advanced Mathematical Methods for Scientists and Engineers (McGraw-Hill, New York, 1978).

[21] R. Zwanzig, Nonequilibrium Statistical Mechanics (Oxford University Press, Oxford, 2001).

[22] R. H. Kraichnan, Phys. Fluids 7, 1723 (1964). 